\title{
INTEGRAL MANIFOLDS OF IMPULSIVE DIFFERENTIAL EQUATIONS ${ }^{1}$
}

\author{
D.D. BAINOV and S.I. KOSTADINOV ${ }^{2}$ \\ Department of Mathematics \\ Plovdiv University \\ BULGARIA \\ and \\ N. VAN MINH, N. HONG THAI, and P.P. ZABREIKO \\ Department of Mathematics \\ Byelorussian University \\ Minsk, USSR
}

\begin{abstract}
The present paper is concerned with the existence of integral manifolds of impulsive differential equations as $t \rightarrow+\infty$. Under the assumption of exponential trichotomy on the linear part of the right-hand side of the equation, it is proved that if the nonlinear perturbation is small enough, then there exist integral manifolds as $t \rightarrow+\infty$ for the perturbed equations.
\end{abstract}

Key words: Integral manifold, exponential trichotomy.

AMS (MOS) subject classification: Primary 34C35.

\section{INTRODUCTION}

Impulsive differential equations have found many applications in physics, chemistry, control theory, etc. In recent years, may interesting results on qualitative theory of these equations have been obtained [1], [2], [5], [10]. The existence of stable and unstable integral manifolds of such equations has been investigated in [10] under the assumption that the linear part of the right-had sides of these equations is hyperbolic. In [2], the authors have investigated the existence of integral manifolds, some components of which remain bounded as $t \rightarrow \pm \infty$.

In this paper we are concerned with the existence of integral manifolds, some components of which remain bounded as $t \rightarrow+\infty$. Introducing the notion of trichotomy of the linear part of the right-hand sides of these equations, we have proved that under a small

\footnotetext{
${ }^{1}$ Received: February, 1991. Revised: March, 1991.
}

${ }^{2}$ Supported by the Bulgarian Ministry of Science \& Higher Education under Grant 61. 
nonlinear perturbation there exist integral manifolds with properties mentioned above for differential equations with impulse effect.

\section{PRELIMINARIES}

In the present paper, we consider equations of the form

$$
\begin{gathered}
\frac{d x}{d t}=A(t) x+F(t, x) \text { if } t \neq t_{n}, t \in \mathbb{R}^{+} \\
\left.\Delta x\right|_{t=t_{n}}=B_{n} x\left(t_{n}-0\right)+I_{n}\left(x\left(t_{n}-0\right)\right), n \in \mathbb{N}
\end{gathered}
$$

where $x \in X, X$ is a complex Banach space, $\left.\Delta x\right|_{t=\tau}=x(\tau+0)-x(\tau-0), F: \mathbb{R}^{+} \times X \rightarrow X$ is a piecewise continuous function with discontinuities of the first kind at the points $t=t_{n}$, $n=1,2, \ldots$ with respect to $t$ and continuous with respect to $x, A(t)$ is a continuous and bounded operator with respect to $t, B_{n}$ are bounded linear operators, $I_{n}: X \rightarrow X$ are impulsive operators. Furthermore,

$$
0<t_{1}<t_{2}<\ldots, \lim _{n \rightarrow \infty} t_{n}=+\infty
$$

We assume that there exists uniformly in $t \in \mathbb{R}^{+}$

$$
\lim _{T \rightarrow+\infty} \frac{i(t, t+T)}{T}=p<+\infty
$$

where $i(a, b)$ is defined as the number of points $t_{n}$ belonging to $(a, b)$.

Definition 1: By solution of (1), we understand a piecewise continuous function $x(t)$, $t \in \mathbb{R}^{+}$with discontinuities of the first kind at the points $t_{n}, n \in N$ such that for every $t \neq t_{n}$ we have

$$
\frac{d x}{d t}=A(t) x(t)+F(t, x(t))
$$

and $x(t)$ satisfies (2) for every $t=t_{n}$.

Definition 2: The linear part of (1) is said to have an exponential trichotomy if there exists a splitting $X=X_{1} \oplus X_{2} \oplus X_{3}$ and positive constants $\kappa, \alpha, \beta, \alpha>\beta$ with the following properties:

i) $\quad A(t), B_{n}$ leave $X_{i}, i=1,2,3$ invariant for every $t \in \mathbb{R}^{+}$and $n \in \mathbb{N}$,

ii) $\quad\left\|X_{2}(t) X_{2}^{-1}(s)\right\| \leq \kappa \exp (-\alpha(t-s))$, for $t \geq s$

$\left\|X_{3}(t) X_{3}^{-1}(s)\right\| \leq \kappa \exp (-\alpha(s-t))$, for $s \geq t$

$\left\|X_{1}(t) X_{1}^{-1}(s)\right\| \leq \kappa \exp (\beta|t-s|)$, for all $t, s$

where $X(t)=\operatorname{diag}\left(X_{1}(t), X_{2}(t), X_{3}(t)\right)$ is a fundamental solution operator of the linear part of 
(1).

Definition 3: We call an integral manifold of (1) any subset $M$ of the extended phase space consisting of whole integral curves.

From now on we shall deal with integral manifolds which can be represented in the form of a graph of a mapping with certain properties.

In what follows $L(\rho, \eta)$ denotes the set of mappings $\varphi: \mathbb{R}^{+} \times X_{3} \rightarrow X_{1} \oplus X_{2}$ enjoying the following properties:

$$
\begin{gathered}
\|\varphi(t, u)\| \leq \rho \text { for }(t, u) \in \mathbb{R}^{+} \times X_{3} \\
\|\varphi(t, u)-\varphi(t, v)\| \leq \eta\|u-v\| \text { for all } t \in \mathbb{R}^{+}, u, v \in X_{3} .
\end{gathered}
$$

We shall consider only nonlinear perturbations $F, I_{n}$ in (1) satisfying the Lipschitz condition with respect to $x$, i.e.

$$
\begin{gathered}
\|F(t, x)-F(t, y)\| \leq \delta\|x-y\| \text { for all } t \in \mathbb{R}^{+}, x, y \in X \\
\left\|I_{n}(x)-I_{n}(y)\right\| \leq \delta\|x-y\| \text { for all } n \in \mathbb{N}, x, y \in X
\end{gathered}
$$

Furthermore, we assume that

$$
\|F(t, x)\| \leq Q,\left\|I_{n}(x)\right\| \leq Q \text { for all } t, n, x .
$$

\section{PREPARATORY LEMMAS}

The proof of our main result needs some technical preparations which we carry out in this section.

Lemma 1: Suppose that the nonlinear perturbations $F, I_{n}$ in (1) are independent of $x$ and $X_{3}=\{0\}$, (i.e., the linear part of (1) has an exponential dichotomy). then (1) has at least one bounded solution. In addition, all bounded solutions of (1) satisfy the following equation

$$
x(t)=X(t) y+\int_{0}^{\infty} G(t, \tau) F(\tau) d \tau+\sum_{i=1}^{\infty} G\left(t, t_{i}\right) I_{i}
$$

where $X(t)$ is the principal fundamental solution operator of the homogeneous equation (i.e., $X(0)=I d), G(t, \tau)$ is Green's function of the homogeneous equation, $y \in X_{2}$.

Proof: The proof of the lemma can be carried out in a well known way (see e.g. [1]). Thus we omit it. 
Lemma 2: Assume that $X_{3}=\{0\}$. Then (1) has at least one bounded solution (as $t \rightarrow+\infty)$ and all bounded solutions of (1) satisfy the equation

$$
x(t)=X(t) y+\int_{0}^{\infty} G(t, s) F(t, x(s)) d s+\sum_{i=1}^{\infty} G\left(t, t_{i}\right) I_{i}\left(x\left(t_{i}\right)\right)
$$

where the notation is the same as in Lemma 1.

It may be noted that under the assumptions stated above equation (1) satisfies the Existence and Uniqueness Theorem.

Now we rewrite equation (1) in another form

$$
\begin{gathered}
\frac{d u}{d t}=A_{1}(t) u+F_{1}(t, u, v), u \in X_{1}, v \in X_{2} \oplus X_{3} \\
\frac{d v}{d t}=A_{2}(t) v+F_{2}(t, u, v), \text { if } t \neq t_{n}, t \in \mathbb{R}^{+} \\
\left.\Delta u\right|_{t=t_{n}}=B_{n}^{1} u\left(t_{n}-0\right)+I_{n}^{1}\left(u\left(t_{n}-0\right), v\left(t_{n}-0\right)\right) \\
\left.\Delta V\right|_{t=t_{n}}=B_{n}^{2} v\left(t_{n}-0\right)+I_{n}^{2}\left(u\left(t_{n}-0\right), v\left(t_{n}-0\right)\right) .
\end{gathered}
$$

Suppose that $M$ is any integral manifold of (5) which is represented by the function $\varphi: \mathbb{R}^{+} \times X_{1} \rightarrow X_{2} \oplus X_{3}, \varphi \in L(\rho, \eta)$. Now we are going to find the equation defining $\varphi$. Let $x(t)$ be any solution of $(1)$ such that $(t, x(t)) \in M$ for all $t \in \mathbb{R}^{+}$. Then $x(t)=(u(t), \varphi(t, u(t))$, where $u(t)=P_{1} \times(t), P_{2}, P_{2}, P_{3}$ denote the projections: $X \rightarrow X_{1}, X_{2}, X_{3}$ respectively. It is clear that $u(t)$ is a solution of the following equation

$$
\begin{gathered}
\frac{d u}{d t}=A_{1}(t) u+F_{1}(t, u, \varphi(t, u)) \text { if } t \neq t_{n} \\
\left.\Delta u\right|_{t=t_{n}}=B_{n}^{1} u+I_{n}^{1}\left(u, \varphi\left(t_{n}, u\right)\right) .
\end{gathered}
$$

On the other hand, $\varphi(t, u(t))$ is a solution of the equation

$$
\begin{gathered}
\frac{d \varphi(t, u(t))}{d t}=A_{2}(t) \varphi(t, u(t))+F_{2}(t, u(t), \varphi(t, u(t))) \text { if } t \neq t_{n}, t \in \mathbb{R}^{+} \\
\left.\Delta \varphi\right|_{t=t_{n}}=I_{n}^{2} \varphi\left(t_{n}, u\left(t_{n}-0\right)\right)+I_{n}^{2}\left(u\left(t_{n}-0\right), \varphi\left(t_{n}, u\left(t_{n}-0\right)\right)\right) .
\end{gathered}
$$

From Lemma 2, it follows that there exists $y \in X_{2}$ such that

$$
\begin{aligned}
\varphi(t, u(t))= & X\left(t, t_{0}\right) y+\int_{t_{0}}^{\infty} G(t, s) F_{2}(s, u(s), \varphi(s, u(s))) d s+ \\
& +\sum_{i=1}^{\infty} G\left(t, t_{i}\right) I_{i}^{2}\left(u\left(t_{i}\right), \varphi\left(t_{i}, u\left(t_{i}\right)\right)\right) .
\end{aligned}
$$


Lemma 3: Suppose that $u(t)$ is piecewise continuous with discontinuities of the first kind at the points $t_{n}, n=1,2, \ldots$ on the interval $\left[t_{0},+\infty\right)$ and satisfies

$$
u(t) \geq \int_{t_{0}}^{t} u(s) v(s) d s+F(t)+\sum_{t_{0}<t_{i}<t} \beta_{i} u\left(t_{i}\right)+\sum_{t_{0}<t_{i}<t} \alpha_{i}(t)
$$

where $v \geq 0$ is piecewise continuous, $\alpha_{i}, F$ are increasing, $\beta_{i} \geq 0$. Then we have

$$
u(t) \leq\left(F(t)+\sum_{t_{0}<t_{i}<t} \alpha_{i}(t)\right) \prod_{t_{0}<t<t}\left(1+\beta_{i}\right) e^{\int_{t_{0}}^{t} v(s) d s} .
$$

Proof: See [2].

Now we assume that $\varphi(t, u, y)$ satisfy the inequality

$$
\left\|\varphi\left(t, u_{1}, y_{1}\right)-\varphi\left(t, u_{2}, y_{2}\right)\right\| \leq \eta\left\|u_{1}-u_{2}\right\|+L e^{-\nu\left(t-t_{0}\right)}\left\|y_{1}-y_{2}\right\|
$$

where $u_{1}, u_{2} \in X_{1} ; y_{1}, y_{2} \in X_{2}, 0<\eta, 0<\nu$.

Let $U(t, s, u \mid \varphi)$ be the solution of $(6)$ corresponding to $\varphi$, starting at the moment $s$ from $u$. We denote

$$
\psi_{1}(t)=U\left(t, s, u_{1} \mid \varphi_{1}\right), \psi_{2}(t)=U\left(t, s, u_{2} \mid \varphi_{2}\right) .
$$

It is easy to verify that for equation (6), we have the variation of parameters formula

$$
\begin{aligned}
\psi_{1}(t)= & X_{1}(t, s) u_{1}+\int_{s}^{t} X_{1}(t, r) F_{1}\left(r, u_{1}, \varphi\left(r, u_{1}, y_{1}\right) d r+\right. \\
& +\sum_{s<t_{i}<t} X_{1}\left(t, t_{i}\right) I_{i}^{1}\left(\psi_{1}\left(t_{i}\right)+\varphi\left(t_{i}, \psi_{1}\left(t_{i}\right)\right)\right)
\end{aligned}
$$

where $X_{1}(t, s)$ is the Cauchy operator of the linear part of (6). So, we have the following estimate for $t \geq s$

$$
\begin{gathered}
\left\|\psi_{1}-\psi_{2}\right\| \leq\left\|X_{1}(t, s)\right\|\left\|u_{1}-u_{2}\right\|+\int_{s}^{t}\left\|X_{1}(t, r)\right\| \| F_{1}\left(r, \psi_{1}(r)+\varphi_{1}\left(r, \psi_{1}(r)\right)\right) \\
-F_{1}\left(r, \psi_{2}(r)+\varphi_{2}\left(r, \psi_{2}(r)\right)\right) \| d r+ \\
+\sum_{s<t_{i}<t}\left\|X_{2}\left(t, t_{i}\right)\right\| \| I_{i}^{1}\left(\psi_{1}\left(t_{i}\right)+\varphi_{1}\left(t_{i}, \psi_{1}\left(t_{i}\right)\right)\right)-I_{i}^{1}\left(\psi_{2}\left(t_{i}\right)+\right. \\
\left.+\varphi_{2}\left(t_{i}, \psi_{2}\left(t_{i}\right)\right)\right) \| \leq
\end{gathered}
$$




$$
\begin{aligned}
& \leq \kappa e^{\beta(t-s)}\left\|u_{1}-u_{2}\right\|+\int_{s}^{t} \kappa e^{\beta(t-r)} \delta\left(\left\|\psi_{1}-\psi_{2}\right\|+\eta\left\|\psi_{1}-\psi_{2}\right\|+\right. \\
& \left.+L e^{-\nu\left(r-t_{0}\right)}\left\|y_{1}-y_{2}\right\|\right) d r+\sum_{s<t_{i}<t} \kappa e^{\beta\left(t-t_{i}\right)} \times \\
& \delta\left(\left\|\psi_{1}\left(t_{i}\right)-\psi_{2}\left(t_{i}\right)\right\|+\eta\left\|\psi_{1}\left(t_{i}\right)-\psi_{2}\left(t_{i}\right)\right\|+L e^{-\nu\left(t_{i}-t_{0}\right)}\left\|y_{1}-y_{2}\right\|\right)
\end{aligned}
$$

where $\varphi_{1}, \varphi_{2}$ denotes $\varphi\left(t, \cdot, y_{1}\right), \varphi\left(t, \cdot, y_{2}\right)$ respectively.

But

$$
\begin{gathered}
u(t)=e^{-\beta t}\left\|\psi_{1}(t)-\psi_{2}(t)\right\|, v(t)=\delta \kappa(1+\eta) \\
F(t)=\kappa e^{-\beta s}\left\|u_{1}-u_{2}\right\|+\delta \kappa L\left\|y_{1}-y_{2}\right\| \int_{s}^{t} e^{-\nu\left(r-t_{0}\right)-\beta r} d r \\
\beta_{i}=(1+\eta) \kappa \delta, \alpha_{i}(t)=\delta \kappa L e^{-(\beta+\nu) t_{i}+\nu t_{0}}\left\|y_{1}-y_{2}\right\|
\end{gathered}
$$

Thus, applying Lemma 3, we obtain for $t \geq s$

$$
\begin{gathered}
\left\|\psi_{1}-\psi_{2}\right\| \leq\left[\kappa e^{\beta(t-s)}\left\|u_{1}-u_{2}\right\|+\right. \\
+\frac{\delta \kappa L}{\beta+\nu} e^{\nu t_{0}}\left\|y_{1}-y_{2}\right\|\left(e^{\beta(t-s)-\nu s}-e^{-\nu t}\right)+ \\
\left.+\sum_{s<t_{i}<t} \delta \kappa L e^{\beta t-(\beta+\nu) t_{i}+\nu t_{0}}\left\|y_{1}-y_{2}\right\|\right](1+(1+\eta) \kappa \delta)^{i(s, t)} e^{\delta \kappa(1+\eta)(t-s)}
\end{gathered}
$$

Now for $t<s$, we have

$$
\begin{gathered}
\left\|\psi_{1}-\psi_{2}\right\| \leq \kappa e^{\beta(s-t)}\left\|u_{1}-u_{2}\right\|+\int_{t}^{s} \kappa e^{\beta(r-t)} \delta\left((1+\eta)\left\|\psi_{1}-\psi_{2}\right\|+\right. \\
\left.+L e^{-\nu\left(r-t_{0}\right)}\left\|y_{1}-y_{2}\right\|\right) d r+\sum_{t<t_{i}<s} \kappa e^{\beta\left(t_{i}-t\right)} \delta\left((1+\eta)\left\|\psi_{1}\left(t_{i}\right)-\psi_{2}\left(t_{i}\right)\right\|+\right. \\
\left.+L e^{-\nu\left(t_{i}-t_{0}\right)}\left\|y_{1}-y_{2}\right\|\right),
\end{gathered}
$$

where $\psi_{1}(t)=\psi\left(t, s, u_{1} \mid \varphi_{1}\right), \psi_{2}(t)=\psi\left(t, s, u_{2} \mid \varphi_{2}\right)$. Thus we get

$$
\begin{aligned}
e^{\beta t}\left\|\psi_{1}(t)-\psi_{2}(t)\right\| \leq \kappa e^{\beta s}\left\|u_{1}-u_{2}\right\|+\int_{t}^{s} \delta \kappa(1+\eta) e^{\beta r}\left\|\psi_{1}(r)-\psi_{2}(r)\right\| d r+ \\
+\delta \kappa \int_{t}^{s} L\left\|y_{1}-y_{2}\right\| e^{-\nu\left(r-t_{0}\right)+\beta r} d r+\sum_{t<t_{i}<s} \delta \kappa(1+\eta) e^{\beta t_{i}}\left\|\psi_{1}\left(t_{i}\right)-\psi_{2}\left(t_{i}\right)\right\|+ \\
+\sum_{t<t_{i}<s} \delta \kappa L e^{-\nu\left(t_{i}-t_{0}\right)+\beta t_{i}}\left\|y_{1}-y_{2}\right\| .
\end{aligned}
$$


Put

$$
\begin{gathered}
u(t)=e^{\beta t}\left\|\psi_{1}(t)-\psi_{2}(t)\right\|, v(t)=\delta \kappa(1+\eta) \\
F(t)=\kappa e^{\beta s}\left\|u_{1}-u_{2}\right\|+\delta \kappa L\left\|y_{1}-y_{2}\right\| \int_{t}^{s} e^{-\nu\left(r-t_{0}\right)+\beta r} d r \\
\beta_{i}=(1+\eta) \kappa \delta, \alpha_{i}(t)=\delta \kappa L e^{(\beta-\nu) t_{i}+\nu t_{0}}\left\|y_{1}-y_{2}\right\|
\end{gathered}
$$

We have the inequality

$$
u(t) \leq F(t)+\int_{t}^{s} u(r) v(r) d r+\sum_{t<t_{i}<s} \beta_{i} U\left(t_{i}\right)+\sum_{t<t_{i}<s} \alpha_{i}(t) .
$$

Note that for $t<s, F$ is nonincreasing. So we have Gronwall's inequality of the same type as for the case $t>s$. Finally we get the estimate for each $t<s$

$$
\begin{gathered}
\left\|\psi\left(t, s, u_{1} \mid \varphi_{1}\right)-\psi\left(t, s, u_{2} \mid \varphi_{2}\right)\right\| \leq\left(\kappa e^{\beta(s-t)}\left\|u_{1}-u_{2}\right\|+\right. \\
\left.+\delta \kappa L\left\|y_{1}-y_{2}\right\| \frac{e^{\nu t_{0}}}{\beta-\nu}\left(e^{\beta(s-t)-\nu s}-e^{-\nu t}\right)+\sum_{t<t_{i}<s} \delta \kappa L e^{-\beta t+(\beta-\nu) t_{i}+\nu t_{0}}\left\|y_{1}-y_{2}\right\|\right) \times \\
\times(1+(1+\eta) \kappa \delta)^{i(t, s)} e^{\delta \kappa(1+\eta)(s-t)}
\end{gathered}
$$

We define $L(\rho, R, \eta, L, \nu, \bar{t})$ as the space of functions $\varphi(t, u, y)\left(t \in\left[t_{0},+\infty\right), u \in X_{1}, y \in X_{2}, \quad\|y\| \leq R\right) \quad$ satisfying $\quad$ condition $\quad(10) \quad$ and $\|\varphi(t, u, y)\| \leq \rho$ for $t \geq \bar{t} \geq t_{0}$

$$
\|\varphi\|\left\|=\sup _{t \geq \bar{t} ; u,\|y\| \leq R}\right\| \varphi(t, u, y) \|
$$

In this space we shall investigate the operator $S$ defined by the formula

$$
\begin{gathered}
(S \varphi)(t, u, y)=X\left(t, t_{0}\right) y+\int_{t_{0}}^{\infty} G(t, s) F_{2}(s, \psi(s, t, u \mid \varphi)+ \\
+\varphi(s, \psi(s, t, u \mid \varphi), y) d s+\sum_{i=1}^{\infty} G\left(t, t_{i}\right) I_{i}^{2}\left(\psi\left(t_{i}, t, u \mid \varphi\right)\right)+ \\
+\varphi\left(t_{i}, \psi\left(t_{i}, t, u \mid \varphi\right)\right)
\end{gathered}
$$

Lemma 4: Let $R, \rho, \eta$ be positive constants, $L>\kappa, 0<\nu<\alpha$. Then there exist $\delta_{0}, Q_{0}$ and $t_{1}>t_{0}$ such that for every $0<\delta<\delta_{0}, 0<Q<Q_{0}, \bar{t}>t_{1}$ the operator $S$ acts in $L(\rho, R, \eta, L, \nu, \bar{t})$. 
Proof: First of all, we shall prove that for $\delta, Q$ small enough, $\bar{t}$ large enough, we have $\|S \varphi\| \| \leq \rho$. In fact,

$$
\begin{gathered}
\|(S \varphi)(t, u, y)\| \leq \kappa e^{-\alpha\left(t-t_{0}\right)}\|y\|+\int_{t_{0}}^{\infty} \kappa e^{-\alpha|t-s|} Q d s+ \\
+\sum_{i=1}^{\infty} \kappa e^{-\alpha\left|t-t_{i}\right|} Q .
\end{gathered}
$$

Since $\left\{t_{n}\right\}$ satisfies condition (2), it is easy to show that

$$
\sum_{i=1}^{\infty} e^{-\alpha\left|t-t_{i}\right|}<C_{\alpha}<+\infty
$$

where $C_{\alpha}$ depends only on $\alpha$ and $\left\{t_{n}\right\}$. Thus if we choose $Q$ small enough, $\bar{t}$ large enough, we get $\|(S \varphi)(t, u, y)\| \leq \rho$ for $\|y\| \leq R, t \in[\bar{t},+\infty), u \in X_{1}$. Now we are going to choose $Q, \delta$ so small that $\widetilde{\varphi}=S \varphi$ satisfies condition (10). We have

$$
\begin{gathered}
\left\|\widetilde{\varphi}\left(t, u_{1}, y_{1}\right)-\widetilde{\varphi}\left(t, u_{2}, y_{2}\right)\right\| \leq \kappa e^{-\alpha\left(t-t_{0}\right)}\left\|y_{1}-y_{2}\right\|+ \\
+\int_{t_{0}}^{\infty} \delta \kappa e^{-\alpha|t-s|}\left((1+\eta)\left\|\psi\left(s, t, u_{1} \mid \varphi_{1}\right)-\psi\left(s, t, u_{2} \mid \varphi_{2}\right)\right\|+\right. \\
\left.+L e^{-\nu\left(s-t_{0}\right)}\left\|y_{1}-y_{2}\right\|\right) d s+ \\
+\sum_{i=1}^{\infty} \delta \kappa e^{-\alpha\left|t-t_{i}\right|}\left((1+\eta)\left\|\psi\left(t_{i}, t, u_{1} \mid \varphi_{1}\right)-\psi\left(t_{i}, t, u_{2} \mid \varphi_{2}\right)\right\|+\right. \\
\left.+L e^{-\nu\left(t_{i}-t_{0}\right)}\left\|y_{1}-y_{2}\right\|\right) .
\end{gathered}
$$

Based on the estimates (12), (13), we obtain

$$
\begin{gathered}
\left\|\tilde{\varphi}\left(t, u_{1}, y_{1}\right)-\tilde{\varphi}\left(t, u_{2}, y_{2}\right)\right\| \leq \kappa e^{-\alpha\left(t-t_{0}\right)}\left\|y_{1}-y_{2}\right\|+ \\
+\int_{t_{0}}^{\infty} \delta \kappa L e^{-\alpha|t-s|-\nu\left(s-t_{0}\right)}\left\|y_{1}-y_{2}\right\| d s+\sum_{i=1}^{\infty} \delta \kappa L e^{-\alpha\left|t-t_{i}\right|-\nu\left(t_{i}-t_{0}\right)}\left\|y_{1}-y_{2}\right\|+ \\
+\int_{t_{0}}^{\infty} \delta \kappa(1+\eta) e^{-\alpha|t-s|}\left\|\psi\left(s, t, u_{1} \mid \varphi_{1}\right)-\psi\left(s, t, u_{2} \mid \varphi_{2}\right)\right\| d s+ \\
+\sum_{i=1}^{\infty} \delta \kappa(1+\eta) e^{-\alpha\left|t-t_{i}\right|}\left\|\psi\left(t_{i}, t, u_{1} \mid \varphi_{1}\right)-\psi\left(t_{i}, t, u_{2} \mid \varphi_{2}\right)\right\| \leq \\
\leq N(\delta)\left\|y_{1}-y_{2}\right\|+\int_{t_{0}}^{t} \delta \kappa(1+\eta) e^{-\alpha(t-s)}\left(\kappa e^{\beta(t-s)}\left\|u_{1}-u_{2}\right\|+\right. \\
\left.+\left\|y_{1}-y_{2}\right\| D\left(e^{\beta(t-s)-\nu t}-e^{-\nu s}\right)+\sum_{s<t_{i}<t} \delta \kappa L e^{-\beta s+(\beta-\nu) t_{i}+\nu t_{0}}\left\|y_{1}-y_{2}\right\|\right) \times
\end{gathered}
$$




$$
\begin{gathered}
\times(1+(1+\eta) \kappa \delta)^{i(s, t)} e^{\delta \kappa(1+\eta)(t-s)} d s+ \\
+\int_{t}^{\infty} \delta \kappa(1+\eta) e^{-\alpha(s-t)}\left(\kappa e^{\beta(s-t)}\left\|u_{1}-u_{2}\right\|+D^{\prime}\left\|y_{1}-y_{2}\right\|\left(e^{\beta(s-t)-\nu t}-\right.\right. \\
\left.\left.-e^{-\nu s}\right)+\sum_{t<t_{i}<s} \delta \kappa L e^{\beta s-(\beta+\nu) t_{i}+\nu t_{0}}\left\|y_{1}-y_{2}\right\|\right)(1+(1+\eta) \kappa \delta)^{i(t, s)} e^{\delta \kappa(1+\eta)(s-t)}+ \\
+\sum_{t_{i}<t} \delta \kappa e^{-\alpha\left(t-t_{i}\right)}\left(\kappa e^{\beta\left(t-t_{i}\right)}\left\|u_{1}-u_{2}\right\|+D\left\|y_{1}-y_{2}\right\| \times\right. \\
\times\left(e^{\beta\left(t-t_{i}\right)-\nu t}-e^{\left.-\nu t_{i}\right)}+\sum_{t_{i}<t_{j}<t} \delta \kappa L e^{-\beta t_{j}+(\beta-\nu) t_{i}+\nu t_{0}}\left\|y_{1}-y_{2}\right\|\right) \times \\
\times(1+(1+\eta) \kappa \delta)^{i\left(t_{i}, t\right)} e^{\delta \kappa(1+\eta)\left(t-t_{i}\right)}+\sum_{t<t_{i}} \delta \kappa(1+\eta) e^{-\alpha\left(t_{i}-t\right)} \times \\
\times\left(\kappa e^{\beta\left(t_{i}-t\right)}\left\|u_{1}-u_{2}\right\|+D^{\prime}\left\|y_{1}-y_{2}\right\|\left(e^{\beta\left(t_{i}-t\right)-\nu t}-e^{\left.-\nu t_{i}\right)+}\right.\right. \\
\quad+\sum_{t<t_{j}<t_{i}}^{\left.\delta \kappa L e^{\beta t_{i}-(\beta+\nu) t}+\nu t_{0}\right)}(1+(1+\eta) \kappa \delta)^{i\left(t, t_{i}\right)} \times \\
\times e^{\delta \kappa(1+\eta)\left(t_{i}-t\right)}
\end{gathered}
$$

where $N(\delta) \rightarrow 0$ as $\delta \rightarrow 0$ and $t_{1}$ is large enough, $D, D^{\prime}$ are positive constants. Put $\delta \kappa(1+\eta)=\gamma$. Then, if $\delta$ is small enough and (2) is satisfied, we have

$$
0<(1+\gamma)^{i(s, t)} e^{\gamma(s-t)}<e^{-\epsilon(s-t)}, 0<\epsilon<\alpha-\beta .
$$

Finally, we get the estimate

$$
\left\|\widetilde{\varphi}\left(t, u_{1}, y_{1}\right)-\widetilde{\varphi}\left(t, u_{2}, y_{2}\right)\right\| \leq M(\delta)\left\|u_{1}-u_{2}\right\|+\kappa(\delta) e^{-\nu\left(t-t_{0}\right)}\left\|y_{1}-y_{2}\right\|
$$

where $M(\delta) \rightarrow 0$ as $\delta \rightarrow 0, \kappa(\delta)<L$ if $\delta$ is small enough and $\kappa<L$. This completes the proof of Lemma 4.

\section{MAIN RESULT}

Theorem 1: Assume that the linear part of (1) has an exponential trichotomy with the above notation. Then for every $\eta, \rho, R, 0<\nu<\alpha$ and $L$ large enough, there exist positive constants $Q_{0}, \delta_{0}, \bar{t}$ with the following properties:

Let $\delta, Q$ belong to $\left(0, \delta_{0}\right),\left(0, Q_{0}\right)$ respectively. Then for every $y \in X_{2},\|y\| \leq R$ equation (1) has an integral manifold represented by a function $\varphi \in L(\rho, \eta)$. In addition, $\varphi$ satisfies the conditions: 
1) $\quad P_{2}(u+\varphi(t, u, y))=y, P_{2}$ is the projection: $X \rightarrow X_{2}$

2) $\quad\|\varphi(t, u, y)\| \leq \rho$ for $t-t_{0} \geq \bar{t},\|y\| \leq R$

3) $\quad\left\|\varphi\left(t, u_{1}, y_{1}\right)-\varphi\left(t, u_{2}, y_{2}\right)\right\| \leq \eta\left\|u_{1}-u_{2}\right\|+l e^{-\nu\left(t-t_{0}\right)}\left\|y_{1}-y_{2}\right\|$.

Proof: From Lemma 4, it follows that there exist positive constants $Q_{0}, \delta_{0}, t_{1}$ such that $S$ acts in $L(\rho, R, \eta, L, \nu, \bar{t})$. Now we are going to show that if $Q_{0}, \delta_{0}$ are chose small enough, then $S$ is contracting. In fact, we have

$$
\begin{gathered}
\left\|(S \varphi)(t, u, y)-\left(S \varphi^{\prime}\right)(t, u, y)\right\| \leq \int_{t_{0}}^{\infty}\|G(t, s)\| \delta(\| \psi(s, t, u \mid \varphi)- \\
\left.\left.-\psi\left(s, t, u \mid \varphi^{\prime}\right)\|+\| \varphi(s, \psi, t, u \mid \varphi)\right)-\varphi^{\prime}\left(s, \psi\left(s, t, u \mid \varphi^{\prime}\right)\right) \|\right) d s \\
+\sum_{i=1}^{\infty}\left\|G\left(t, t_{i}\right)\right\| \delta\left(\left\|\psi\left(t_{i}, t, u \mid \varphi\right)-\psi\left(t_{i}, t, u \mid \varphi^{\prime}\right)\right\|+\right. \\
\left\|\varphi\left(t_{i}, \psi^{\prime}\left(t_{i}, t, u \mid \varphi\right)\right)-\varphi^{\prime}\left(t_{i}, \psi\left(t_{i}, t, u \mid \varphi^{\prime}\right)\right)\right\|
\end{gathered}
$$

where $\psi(t, s, u \mid \varphi)$ is the solution of $(6)$.

On the other hand, we have

$$
\begin{gathered}
\left\|\psi(s, t, u \mid \varphi)-\psi\left(s, t, u \mid \varphi^{\prime}\right)\right\| \leq \int_{t}^{s} \kappa \delta e^{\beta(s-\xi)}\left(\left\|\psi_{1}-\psi_{2}\right\|+\left\|\varphi-\varphi^{\prime}\right\| \mid\right) d \xi \\
+\sum_{t<t_{i}<s} \delta \kappa e^{\beta\left(s-t_{i}\right)}\left(\left\|\psi_{1}\left(t_{i}\right)-\psi_{2}\left(t_{i}\right)\right\|+\left\|\varphi-\varphi^{\prime}\right\| \mid\right) .
\end{gathered}
$$

Setting $u(s)=e^{-\beta s}\left\|\psi_{1}(s)-\psi_{2}(s)\right\|$ and applying Gronwall's inequality, we have

$$
\begin{gathered}
u(s) \leq\left(\int_{t}^{s} \kappa \delta e^{-\beta \xi} d \xi+\sum_{t<t_{i}<s} \kappa \delta e^{-\beta t_{i}}\right)\left\|\varphi-\varphi^{\prime}\right\| \times \times \\
(1+\delta \kappa)^{i(t, s)} e^{\delta \kappa(s-t)} .
\end{gathered}
$$

So we have

$$
\left\|\psi(s, t, u \mid \varphi)-\psi^{\prime}\left(s, t, u \mid \varphi^{\prime}\right)\right\| \leq D(\delta) e^{\beta(s-t)}\|\| \varphi-\varphi^{\prime}\|\|
$$

where $D(\delta) \rightarrow 0$ as $\delta \rightarrow 0$.

Substituting (19) into (17), we have

$$
\begin{gathered}
\left\|(S \varphi)(t, u, y)-\left(S \varphi^{\prime}\right)(t, u, y)\right\| \leq\left[\delta \int_{t_{0}}^{\infty} \kappa e^{-\alpha|t-s|}\left(D(\delta)^{\beta|s-t|}+1\right) d s\right. \\
\left.+\sum_{i=1}^{\infty} \kappa \delta e^{-\alpha\left|t-t_{i}\right|}\left(D(\delta) e^{\beta\left|t_{i}-t\right|}+1\right)\right]\left\|\left|\varphi-\varphi^{\prime}\right|\right\| .
\end{gathered}
$$


From (2), (20) it follows that if $\delta$ is sufficiently small, then $S$ is contracting. This completes the proof of the theorem.

\section{REFERENCES}

[1] D.D. Bainov, S.I. Kostadinov, P.P. Zabreiko, "Exponential dichotomy of linear impulsive equations in a Banach space”, Intern. J. Theor. Phys., 28, No. 7, (1989), pp. 797-814.

[2] D.D. Bainov, S.I. Kostadinov, N. Hong Thai, P.P. Zabreiko, "Existence of integral manifolds for impulsive differential equations in a Banach space", Intern. J. Theor. Phys., 28, No. 7, (1989), pp. 815-833.

[3] T.G. Bychkova, "Integral manifolds and N.N. Bogoljubov's method of averaging", in Qualitative and Approximation Methods of Investigation of Operator Equations, ed. by P.P. Zabreiko, Jaroslav, (1978), pp. 30-47.

[4] Ju. L. Daleckii, M.G. Krein, "Stability of Solutions of Differential Equations in Banach Space", Nauka, Moscow, (1970), (in Russian).

[5] V. Lakshmikantham, D.D. Bainov, P.S. Simeonov, "Theory of Impulsive Differential Equations", World Scientific, Singapore, (1989).

[6] V.D. Mil'man, A.D. Myshkis, "Stability of motion in the presence of impulses", Sib. Math. J., 1, No. 2, (1960), pp. 233-237, (in Russian).

[7] V.D. Mil'man, A.D. Myshkis, "Stochastic impulses in linear dynamical systems", Approx. Methods of Solution of Differential Equations, Kiev, (1963), pp. 64-81, (in Russian).

[8] Ju. A. Mitropolskii, O.B. Lykova, “Integral Manifolds in Nonlinear Mechanics", Nauka, Moscow, (1973), (in Russian).

[9] A.D. Myshkis, A.M. Samoilenko, "Systems with impulses at fixed moments", Mat. Sb., 74, No. 2, (1967), pp. 202-208, (in Russian).

[10] A.M. Samoilenko, N.A. Perestyuk, "Differential Equations with Impulse Effect", Visca Skola, Kiev, (1987), (in Russian). 


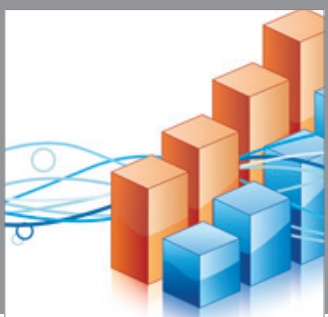

Advances in

Operations Research

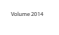

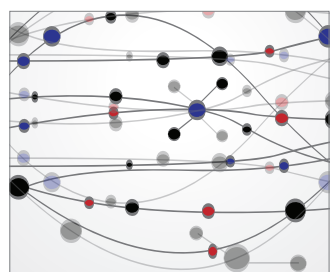

\section{The Scientific} World Journal
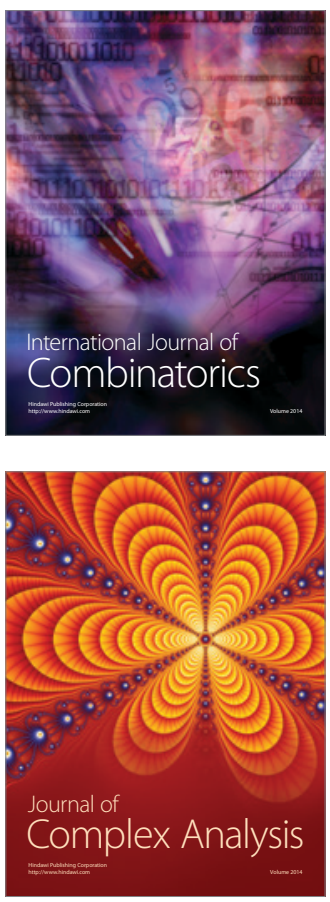

International Journal of

Mathematics and

Mathematical

Sciences
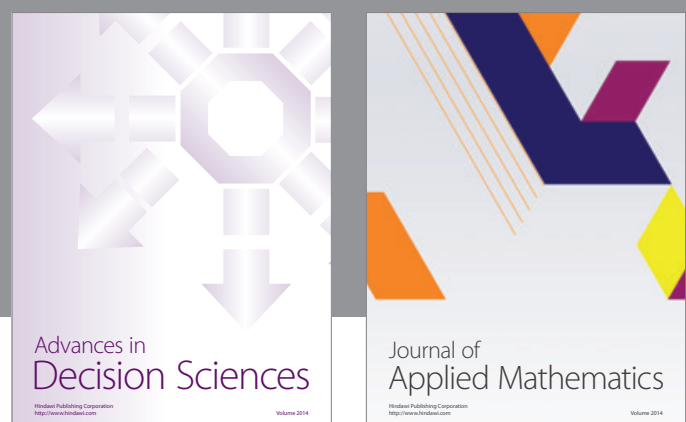

Journal of

Applied Mathematics
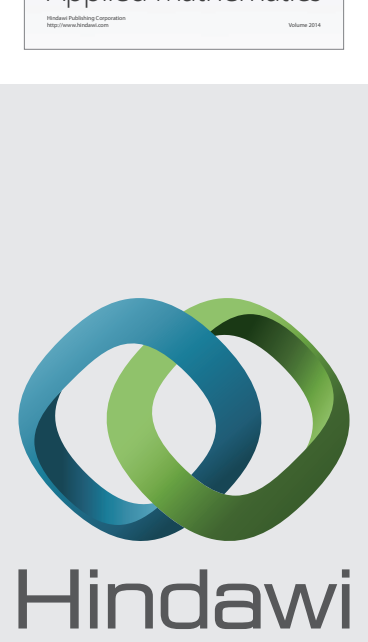

Submit your manuscripts at http://www.hindawi.com
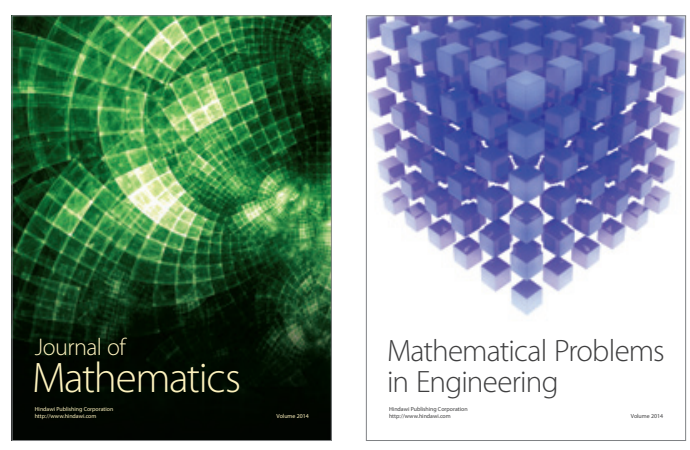

Mathematical Problems in Engineering
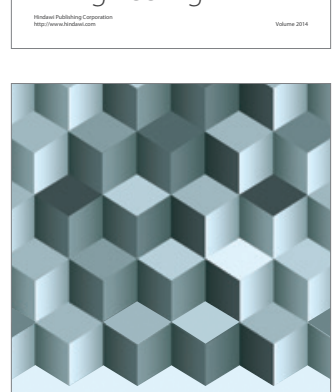

Journal of

Function Spaces
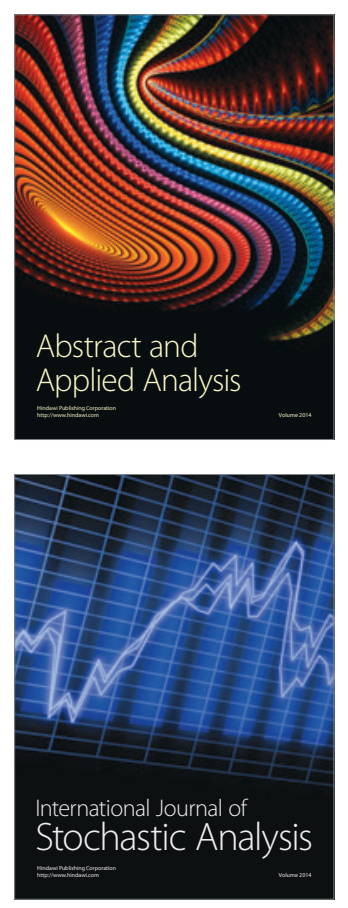

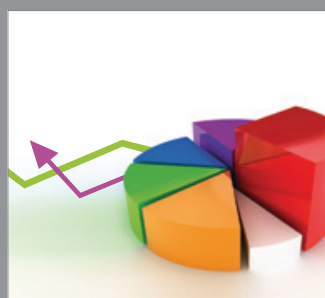

ournal of

Probability and Statistics

Promensencen
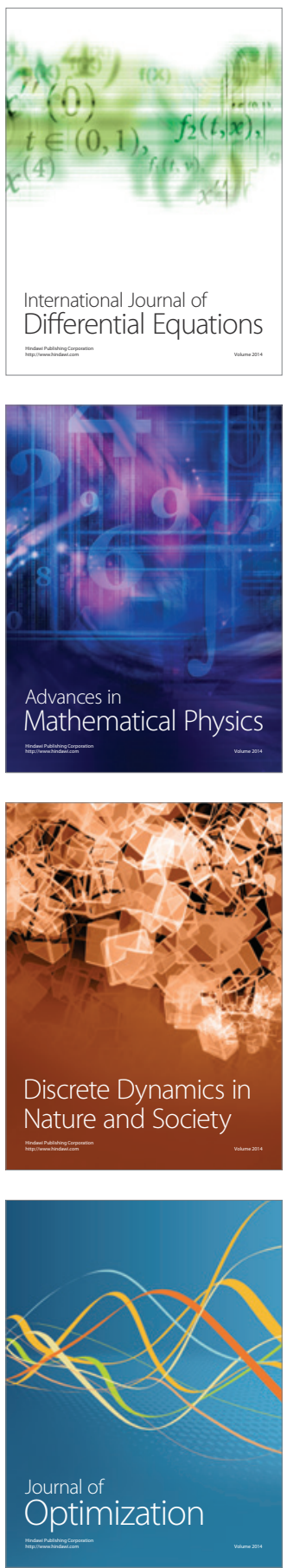